\title{
Bacterial partitioning proteins affect the subcellular location of broad-host-range plasmid RK2
}

\author{
Katarzyna Kolatka, Monika Witosinska, Marcin Pierechod \\ and Igor Konieczny
}

Correspondence

Igor Konieczny

igor@biotech.univ.gda.pl

Received 22 March 2008

Revised 28 May 2008

Accepted 5 June 2008

\section{Department of Molecular and Cellular Biology, Intercollegiate Faculty of Biotechnology, University of Gdansk, Kladki 24, 80-822 Gdansk, Poland}

\begin{abstract}
It has been demonstrated that plasmids are not randomly distributed but are located symmetrically in mid-cell, or $1 / 4,3 / 4$ positions in bacterial cells. In this work we compared the localization of broad-host-range plasmid RK2 mini-replicons, which lack an active partitioning system, in Escherichia coli and Pseudomonas putida cells. In E. coli the location of the plasmid mini-replicon cluster was at the cell poles. In contrast, in Pseudomonas cells, as a result of the interaction of chromosomally encoded ParB protein with RK2 centromere-like sequences, these miniderivatives were localized in the proximity of mid-cell, or $1 / 4,3 / 4$ positions. The expression of the Pseudomonas parAB genes in E. coli resulted in a positional change in the RK2 mini-derivative to the mid-cell or $1 / 4,3 / 4$ positions. Moreover, in a $P$. putida parAB mutant, both RK2 miniderivatives and the entire RK2 plasmid exhibited disturbances of subcellular localization. These observations raise the possibility that in certain bacteria chromosomally encoded partitioning machinery could affect subcellular plasmid positioning.
\end{abstract}

\section{INTRODUCTION}

Spatial organization, localization and appropriate distribution of genetic material are essential for all cellular organisms. Major advances in the understanding of DNA dynamics in bacteria have been achieved by using fluorescence microscopy to visualize the intracellular location of plasmids and bacterial chromosomes. These studies have shown that plasmids are not randomly distributed but are located at distinct sites within bacterial cells. The low-copy-number Escherichia coli plasmids P1 (Gordon et al., 1997; Li \& Austin, 2002) and F (Gordon et al., 1997, 2004; Niki \& Hiraga, 1997) as well as Salmonella enterica plasmid R27 (Lawley \& Taylor, 2003) are found at the mid-cell of newborn cells or symmetrically at the $1 / 4$ and $3 / 4$ positions in older, pre-divisional cells. For some plasmids, it was proposed that plasmid particles could form clusters. Plasmid R1, with a copy number of four to five per cell, also forms symmetrically located clusters, although in older cells, R1 clusters are shifted towards the cell poles (Jensen \& Gerdes, 1999; Weitao et al., 2000). The mid-cell, $1 / 4$ and $3 / 4$ cellular positioning of plasmid clusters has been observed for the broad-hostrange plasmid RK2 (60 kb in size) in E. coli, Pseudomonas aeruginosa and Vibrio cholerae (Pogliano et al., 2001; Ho et al., 2002). This plasmid belongs to the IncP-1 incompatibility group, is present at five to eight copies per chromosome, and is stably maintained in almost all

Abbreviations: DAPI, 4',6-diamidino-2-phenylindole; FISH, fluorescence in situ hybridization.
Gram-negative bacteria (Rosche et al., 2000; Thomas et al., 1980, 1984; Wilson et al., 1997). When plasmids RK2, F and P1 were localized simultaneously, the majority of plasmid foci were close to but separated from each other, demonstrating that compatible bacterial plasmids are in independent cellular locations in E. coli (Ho et al., 2002). The subcellular localization of these plasmids is thought to be mediated by the partitioning (Par) systems they encode. Plasmid partitioning loci function as a cassette containing genes for two trans-acting proteins and a cis-acting centromere-like site. The first gene of a par operon encodes a ParA-like protein, which is a Walker box or actin-like ATPase (Ebersbach \& Gerdes, 2005; Funnell, 2005). The product of the second gene, a ParB-like protein, binds as a dimer to specific sequences within the cis site (Schumacher \& Funnell, 2005). The formation of a partitioning complex and oscillations of the filamentforming ParA ATPase were proposed to result in localization of the plasmid to the mid-cell or the $1 / 4$ and $3 / 4$ positions, which represent the middle of the next cell generation (Ebersbach \& Gerdes, 2004; Ebersbach et al., 2006). For plasmid RK2, the partitioning cassette consists of the genes encoding IncC, a Walker box ATPase, and $\mathrm{KorB}$, a protein that interacts with a palindromic operator $\left(\mathrm{O}_{\mathrm{B}}\right)$ found 12 times in RK2 DNA (Kostelidou \& Thomas, 2000; Rosche et al., 2000; Williams et al., 1998). The rules governing plasmid positioning in bacterial cells are not fully understood. It is not known, for example, what bacterial factors affect plasmid cluster formation, localization and partitioning. In this work we demonstrate that 
chromosomal partitioning genes affect the subcellular location of a plasmid mini-replicon.

\section{METHODS}

Plasmids and bacterial strains. The plasmids and bacterial strains used in this work are listed in Table 1. Plasmids were introduced into $E$. coli and Pseudomonas cells by transformation (Sambrook et al., 1989).

Media and growth conditions. Bacteria were grown at $30{ }^{\circ} \mathrm{C}$ in $\mathrm{LB}$ or M63/glucose media. Antibiotics were added to the following final concentrations: ampicillin $50 \mu \mathrm{g} \mathrm{ml}^{-1}$ for liquid media or $100 \mu \mathrm{g}$ $\mathrm{ml}^{-1}$ for agar plates, chloramphenicol up to $150 \mu \mathrm{g} \mathrm{ml} \mathrm{g}^{-1}$, kanamycin $30 \mu \mathrm{g} \mathrm{ml}^{-1}$ for liquid media or $60 \mu \mathrm{g} \mathrm{ml}^{-1}$ for agar plates, gentamicin up to $20 \mu \mathrm{g} \mathrm{ml}^{-1}$, tetracycline up to $50 \mu \mathrm{g} \mathrm{ml}^{-1}$.

Fluorescence in situ hybridization (FISH). Mini-RK2 specific Cy3-labelled probes for FISH were prepared essentially as described by Pogliano et al. (2001). The procedure was modified as follows. After incubation with the blocking solution, the cells were treated with lysozyme ( $1 \mathrm{mg} \mathrm{ml}^{-1}$ in PBS) for $10 \mathrm{~min}$ at room temperature. The next step was the same as described by Jensen \& Shapiro (1999). Chromosomal DNA was stained with 4',6-diamidino-2-phenylindole (DAPI) at a concentration of $0.5 \mu \mathrm{g} \mathrm{ml}^{-1}$.

Fluorescence microscopy. E. coli S17-1 and Pseudomonas strains were prepared for microscopy as described previously (Ho et al., 2002; Pogliano et al., 2001). The expression of tetR-eyfp from pKO10 and pKO15 was induced with $0.4 \%$ arabinose for $1 \mathrm{~h}$. The expression of parA, parB and tetR-eyfp from pLAK15 in E. coli cells was induced with $0.4 \%$ arabinose for $1.5 \mathrm{~h}$. The expression of tetR-eyfp in E. coli MGTRY was induced as described by Verheust \& Helinski (2007). The cells were stained with FM4-64 $\left(0.5 \mu \mathrm{g} \mathrm{ml}^{-1}\right)$, immobilized on a poly-L-lysine-treated slide coverslip and observed using an Olympus BX51 fluorescence microscope with F-View-II CCD camera. Measurements and image analysis were conducted with AnalySIS software.

Gel filtration assay. Column gel filtration was used to fractionate the DNA-ParB complexes as described previously (Konieczny \& Helinski, 1997). The reaction mixture $(100 \mu \mathrm{l})$, containing $P$. putida ParB $(4 \mu \mathrm{g})$ and the indicated supercoiled plasmid DNA $(2 \mu \mathrm{g})$ in $40 \mathrm{mM}$ HEPES/KOH, $\mathrm{pH}$ 8.0, $40 \mathrm{mM}$ potassium glutamate, $10 \mathrm{mM}$ magnesium acetate, $4 \%$ sucrose, $4 \mathrm{mM}$ dithiothreitol, and $2 \mathrm{mM}$ ATP, was incubated for $20 \mathrm{~min}$ at $32{ }^{\circ} \mathrm{C}$ and then run through a Sepharose CL-4B $(0.5 \times 12 \mathrm{~cm})$ column, equilibrated at room temperature with the incubation buffer and $0.01 \%$ Brij 58 . Fractions $(80 \mu \mathrm{l})$ were collected and a portion of each $(35 \mu \mathrm{l})$ was analysed by SDS-PAGE followed by silver staining, while another portion $(35 \mu \mathrm{l})$ was analysed on agarose gel stained with ethidium bromide.

Formaldehyde cross-linking and chromatin immunoprecipitation. Cells were grown at $30{ }^{\circ} \mathrm{C}$ in $\mathrm{LB}$ medium. Samples were taken during exponential growth $\left(\mathrm{OD}_{600} 0.6\right)$ and prepared as described by Lin \& Grossman (1998). Protein-DNA complexes were immunoprecipitated with polyclonal anti-ParB antibodies (C. M. Thomas, University of Birghmingam, UK), followed by incubation with $30 \mu 1$ of a $50 \%$ Protein A-Sepharose slurry (Amersham Pharmacia). PCR was performed with Taq DNA polymerase (Fermentas) and oligonucleotide primers $\left(\mathrm{O}_{\mathrm{B}}{ }^{3}, 5^{\prime}\right.$-TCGCCGTTGCGAACCACCTTCGG-3' 5' $^{\prime}$ AAATCGGGAGTGCGAAAAGCATCACC-3'; ${ }^{10}{ }^{10}, 5^{\prime}$-GATTATGGCTCATATCGAAAGTCTC-3', 5 '-GAGCACACGAAGGATGTTGGTG-3'; oriV, 5'-AAGCCGTGTGCGAGACACCGC-3'， 5' AAAGACAGGTTAGCGGTGGC-3'; IR-2, 5'-CCGCTTCAGCTCA-
TCAACCCAGAC-3', 5' -CTTGCGAAGCTGGGTCAAGTGTACC-3'). PCR products were separated on agarose gels and stained with ethidium bromide.

Purification of ParB. The P. putida ParB protein was expressed from plasmid pETK1 (Table 1). The bacterial strain, the conditions of parB expression and the protein purification were the same as described for the P1 plasmid ParB homologue (Davis \& Austin, 1988) with the exception that only a phosphocellulose column was used. Peak fractions were pooled and dialysed against $\mathrm{P}$ buffer $(150 \mathrm{mM} \mathrm{NaCl})$. The final product yielded $8 \mu \mathrm{g}^{-1}$ of $>95 \%$ homogeneous protein.

Plasmid stabilization assays. The cells carrying a mini-RK2 (pCVI) or RK2 (pZZ15) plasmid were diluted from overnight cultures into LB medium containing appropriate antibiotics (see above, Media and growth conditions) and incubated to $\mathrm{OD}_{600}$ 0.5. The cells were then diluted into medium without antibiotics to allow free growth of RK2 and mini-RK2 segregants and maintained in exponential growth for at least 120 generations by sequential dilutions. The samples removed during this period were plated on agar plates and, from each plating, $\geqslant 400$ colonies were patched onto agar with antibiotics to score retention or loss of plasmids. The number of generations was estimated from optical density measurements.

\section{RESULTS AND DISCUSSION}

\section{The subcellular position of RK2 mini-replicons depends on the host bacterium}

To test for the possibility that a host cell can affect plasmid positioning, a dual plasmid system was used allowing identification of the location of clusters of the miniderivative pCVI of broad-host-range plasmid RK2 in the cells of different host bacteria. pCVI, which lacks partitioning genes but contains two centromere-like sites $\left(\mathrm{O}_{\mathrm{B}}{ }^{3}\right.$ and $\left.\mathrm{O}_{\mathrm{B}}{ }^{10}\right)$, the gene for plasmid replication initiator protein $(\operatorname{trf} A)$, the origin for vegetative DNA replication (oriV), the origin for conjugational transfer (oriT), antibiotic resistance determinants and a tet $O$ array, was tagged by the TetR-EYFP fusion protein expressed from pKO10, an RK2-compatible dual-origin broad-host-range plasmid. The subcellular location of pCVI was examined after arabinose induction of the tetR-eyfp gene and staining of the cell membrane with the fluorescent dye FM4-64. When both plasmids were introduced into E. coli cells, arabinose induction resulted in fluorescent signals mainly localized at one of the cell poles (Fig. 1A i, ii, iii; D). Fluorescent foci were observed in only approximately $50 \%$ of the cells, the majority of which had only one focus. A small fraction of E. coli cells had two foci (Fig. 1A iv, E). A similar subcellular distribution of pCVI was obtained when the TetR-EYFP protein was expressed from the tetR-eyfp gene on the chromosome of E. coli MGTRY (data not shown). Polar positioning of pCVI was confirmed by FISH in E. coli cells containing only this plasmid (Fig. 1B). Plasmid fluorescent signals were observed on the edge of DAPI-stained E. coli nucleoids, suggesting that the plasmid was not located in the mid-cell or $1 / 4,3 / 4$ positions but at the cell poles. Similarly as we observed for pCVI, it was previously reported that $\mathrm{F}$ and $\mathrm{R} 1$ mini-derivatives lacking 
Table 1. Bacterial strains and plasmids

\begin{tabular}{|c|c|c|c|}
\hline Strain or plasmid & Relevant characteristics & Resistance & Reference or source \\
\hline \multicolumn{4}{|l|}{ Strains } \\
\hline E. coli S17-1 & pro hsdR recA with integrated Tral region of plasmid RP4 (Tc::Mu-Spc::Tn7) & $\mathrm{Spc}^{\mathrm{R}}$ & MacKenzie et al. (1995) \\
\hline E. coli MGTRY & $\begin{array}{l}\text { MG1655 ( } \mathrm{F} \lambda^{-} i l v G r f b-50 r p h-1 \text { ) derivative containing gene for fusion protein TetR-EYFP under the control } \\
\text { of } \mathrm{P}_{\mathrm{BAD}} \text { promoter }\end{array}$ & $\mathrm{Spc}^{\mathrm{R}}$ & Verheust \& Helinski (2007) \\
\hline P. aeruginosa PAO1161 & [PAO001] pyrB49 chl-2 & - & Isaac \& Holloway (1968) \\
\hline P. putida KT2440 & $\mathrm{pWW}^{-}$Res $^{-}$derivative of $P$. putida $\mathrm{mt}-2$ & - & Franklin et al. (1981) \\
\hline $\begin{array}{l}\text { P. putida KT2440 } \\
\triangle \text { parAB }\end{array}$ & P. putida KT2440 derivative, $\triangle$ parAB $\Omega$ kan & $\operatorname{Kan}^{\mathrm{R}}$ & $\begin{array}{l}\text { Godfrin-Estevenon et al. } \\
\text { (2002) }\end{array}$ \\
\hline \multicolumn{4}{|l|}{ Plasmids } \\
\hline RK2 & Broad-host-range IncP plasmid & $A m p^{R}, \operatorname{Kan}^{\mathrm{R}}, \mathrm{Tet}^{\mathrm{R}}$ & Meyer et al. (1975) \\
\hline pZZ15 & RK2 derivative containing $l a c O$ array and gene for fusion protein LacI-GFP & $A m p^{R}$, Tet $^{R}$ & Ho et al. (2002) \\
\hline pCVI & Mini-RK2 ( $\mathrm{pFF} 1$ derivative) containing tet $O$ array, $t f r A$ gene, oriV and oriT & $\mathrm{Cm}^{\mathrm{R}}, \mathrm{Gent}^{\mathrm{R}}$ & Verheust \& Helinski (2007) \\
\hline pLAU53 & pUC18 derivative containing genes for fusion proteins LacI-ECFP and TetR-EYFP & $\mathrm{Amp}^{\mathrm{R}}$ & Lau et al. (2003) \\
\hline pLAK53 & $\begin{array}{l}\text { pUC18 derivative containing gene for fusion protein TetR-EYFP, constructed by NcoI deletion of plasmid } \\
\text { pLAU53 }\end{array}$ & $A m p^{R}$ & This work \\
\hline pLAK15 & $\begin{array}{l}\text { pLAK53 derivative containing gene for fusion protein TetR-EYFP and genes for ParA and ParB partitioning } \\
\text { proteins from } P \text {. putida under the control of } \mathrm{P}_{\mathrm{BAD}} \text { promoter }\end{array}$ & $A m p^{R}$ & This work \\
\hline pLAK16 & $\begin{array}{l}\text { pLAK15 derivative containing 'parAparB' fragment from } P \text {. putida and deletion of gene for fusion protein } \\
\text { TetR-EYFP }\end{array}$ & $\mathrm{Amp}^{\mathrm{R}}$ & This work \\
\hline pBBR1MCS-2 & Broad-host-range cloning vector & $\operatorname{Kan}^{\mathrm{R}}$ & Kovach et al. (1995) \\
\hline pKO10 & $\begin{array}{l}\text { Dual-origin (pUC-pBBR1MCS-2) broad-host-range cloning vector; entire pLAK53 inserted into HindIII } \\
\text { site of vector pBBR1MCS-2 }\end{array}$ & $\mathrm{Kan}^{\mathrm{R}}, \mathrm{Amp}^{\mathrm{R}}$ & This work \\
\hline pKO15 & $\begin{array}{l}\text { Dual-origin (pUC-pBBR1MCS-2) broad-host-range cloning vector; 'gidBparB'parA' fragment (2792 bp) } \\
\text { from P. putida (Godfrin-Estevenon et al., 2002) inserted into HindIII and BamHI sites of pKO10 }\end{array}$ & $\operatorname{Kan}^{\mathrm{R}}, A m p^{\mathrm{R}}$ & This work \\
\hline pKO16 & Dual-origin (pUC-pBBR1MCS-2) broad-host-range cloning vector, constructed by ClaI deletion of pKO15 & $\mathrm{Kan}^{\mathrm{R}}$ & This work \\
\hline pUC18-IR2 & $\begin{array}{l}\text { pUC18 derivative; insertion of fragment with IR-2 sequence from } P \text {. putida chromosome and Mph1103 site } \\
\text { (5'-ctagAGGTTCCACGTGGAACAATGCATC; } 5^{\prime} \text {-ccggGATGCATTGTTCCACGTGGAACCT) into XbaI } \\
\text { and Eco88I sites of vector pUC18 }\end{array}$ & $A m p^{R}$ & This work \\
\hline $\mathrm{pUC} 18-\mathrm{O}_{\mathrm{B}}{ }^{3}$ & $\begin{array}{l}\text { pUC18 derivative; insertion of fragment with } \mathrm{O}_{\mathrm{B}}{ }^{3} \text { sequence from RK2 plasmid and Mph1103 site }\left(5^{\prime} \text { - }\right. \\
\text { ctagACCCTTTTTAGCCCGCTAAAACTCTATGCAT; } 5^{\prime} \text {-ccggGATGCATAGAGTTTTAGCGGGCTAAAA- } \\
\text { AGGGT) into XbaI and Eco88I sites of vector pUC18 }\end{array}$ & $\mathrm{Amp}^{\mathrm{R}}$ & This work \\
\hline $\mathrm{pUC18- \textrm {O } _ { \mathrm { B } }}{ }^{10}$ & $\begin{array}{l}\text { pUC18 derivative; insertion of fragment with } \mathrm{O}_{\mathrm{B}}{ }^{10} \text { sequence from RK2 plasmid and } M p h 1103 \text { site }\left(5^{\prime} \text { - }\right. \\
\text { ctagAAGAACTTTAGCGGGCTAAAATTTATGCATC; } 5^{\prime} \text {-ccggGATGCATAAATTTTAGCCCGCTAAAGTTCTT) } \\
\text { into XbaI and Eco88I sites of vector pUC18 }\end{array}$ & $\mathrm{Amp}^{\mathrm{R}}$ & This work \\
\hline pETK1 & $\begin{array}{l}\text { pET22b (Novagen) derivative with deletion of His-tag sequence and insertion gene for ParB protein from } \\
\text { P. putida into NdeI and BamHI sites }\end{array}$ & $A m p^{R}$ & This work \\
\hline
\end{tabular}




\section{E. coli}

A

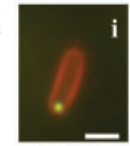

B

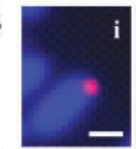

C
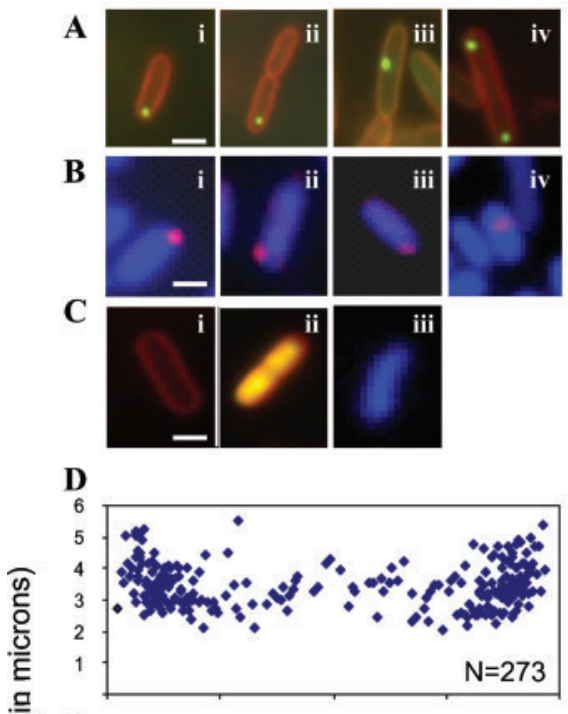

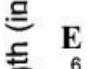

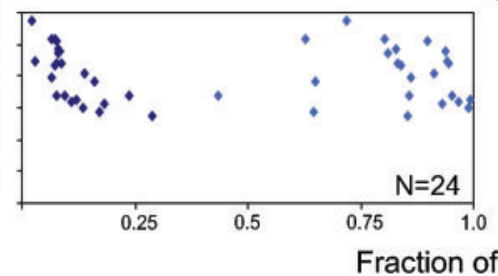

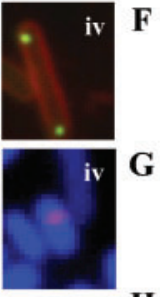

H
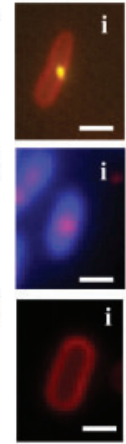

I

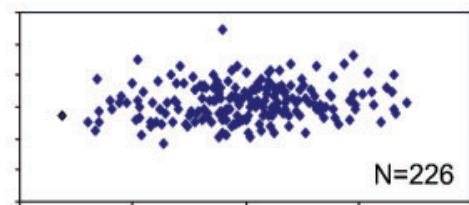

$\mathbf{J}$

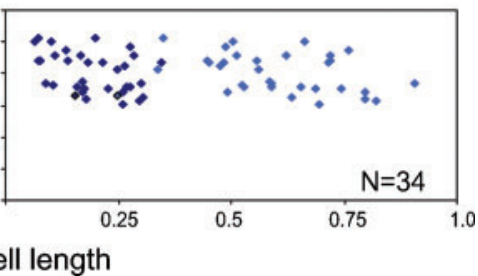

\section{P. putida}
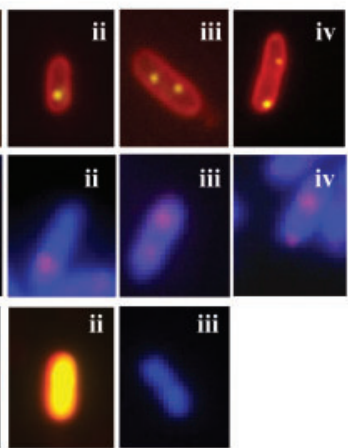

iii

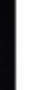
P. putida $(\mathrm{I}, \mathrm{J})$ for cells containing one focus $(\mathrm{D}$, relative position of a single focus measured as the distance to the pole in relation to the cell length. The pole was chosen randomly. Cell length is plotted against position of foci given as a fraction of cell length. Negative controls for tagging with TetR-EYFP and FISH are shown in panels $\mathrm{C}$ and $\mathrm{H}$ : representative $E$. coli S17-1 and P. putida KT2440 cells containing just pCVI (C i, $\mathrm{H}$ i), pKO10 (C ii, $\mathrm{H}$ ii) or no plasmid DNA (C iii, $\mathrm{H}$ iii).

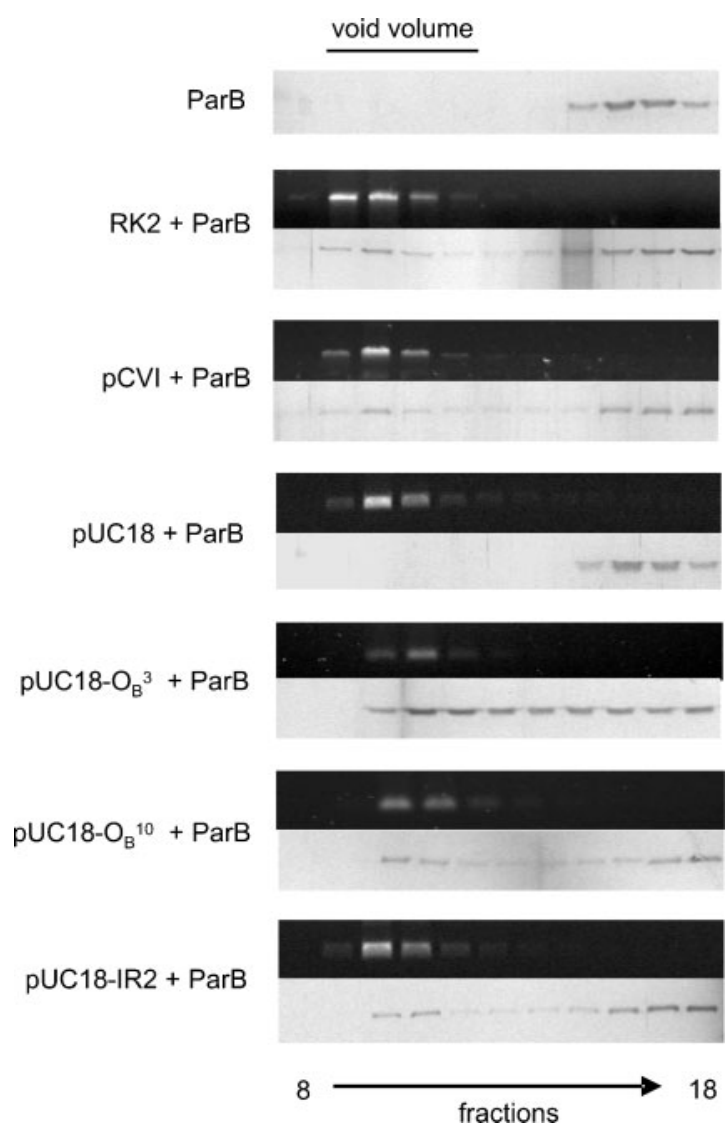

the partitioning segments are distributed at the cell poles (Ebersbach \& Gerdes, 2004; Niki \& Hiraga, 1997). Polar localization of RK2 mini-replicons in E. coli cells has also been reported recently by Verheust \& Helinski (2007), who showed that insertion of the RK2 par locus into pCVI restores the mid-cell, or $1 / 4,3 / 4$ positions of plasmid foci.

When we examined the localization of pCVI in P. putida, the results differed substantially from those obtained in $E$. coli. Instead of a polar location, we observed that the plasmid formed foci at approximately mid-cell and the $1 / 4$, $3 / 4$ positions (Fig. $1 \mathrm{~F} \mathrm{i,} \mathrm{J).} \mathrm{Also} \mathrm{FISH} \mathrm{and} \mathrm{DAPI} \mathrm{co-staining}$ demonstrated plasmid foci inside DAPI-stained P. putida nucleoids (Fig. 1G). Overall, the location of pCVI foci in Pseudomonas cells was similar to the location of intact RK2 (data not shown), although with a broader distribution in the case of the mini-derivative clusters. Also, as observed in E. coli, foci of the mini-derivative were detected in only a

Fig. 2. ParB protein from $P$. putida forms a complex with plasmid RK2 and its mini-derivative in vitro. The reaction mixture contained the indicated supercoiled DNA (RK2, pCVI, pUC18, pUC18$\mathrm{O}_{\mathrm{B}}{ }^{3}{ }_{\text {(RK2) }}, \quad \mathrm{pUC18}-\mathrm{O}_{\mathrm{B}}{ }^{10}{ }_{(\mathrm{RK} 2)}, \quad \mathrm{pUC18}-\mathrm{IR} 2_{(P \text {. putida) })}$ and ParB protein in the amounts and with the incubation conditions described in Methods. After incubation, the reaction mixtures were loaded on a Sepharose CL-4B column. Fractions were collected and analysed by SDS-PAGE followed by silver staining. Agarose gels were stained with ethidium bromide. 

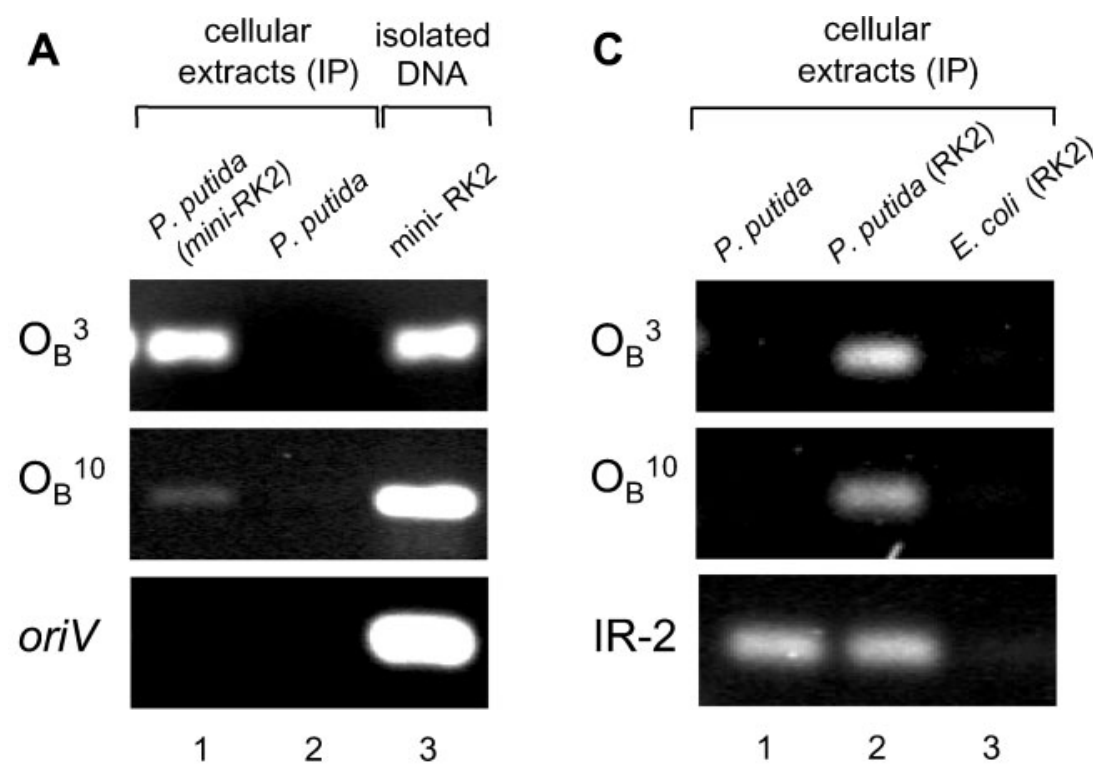

Fig. 3. ParB interacts in live P. putida KT2440 cells with centromere-like sites of plasmid RK2 and its mini-derivative. The PCR-amplified products of immunoprecipitation (IP) with anti-ParB antibodies and lysates of $P$. putida KT2440 (A lane 2; B lane 2, C lane 1), $P$. putida KT2440(pCVI) (A lane 1, B lane 1) or $P$. putida KT2440(RK2) (C lane 2). Lanes 3, control reactions with isolated DNA $(A, B)$ and negative control of immunoprecipitation with anti-ParB antibodies and E. coli S17-1(RK2) lysates (C). The PCR-amplified products were separated on $1 \%$ agarose gels and stained with ethidium bromide. Primers used in reactions were designed for RK2 plasmid sequences containing $\mathrm{O}_{\mathrm{B}}{ }^{3}, \mathrm{O}_{\mathrm{B}}{ }^{10}$ and oriv regions and the IR-2 sequence of $P$. putida KT2440 chromosomal DNA as indicated. All primer sequences are listed in Methods.

fraction of the Pseudomonas cells (60\%). Our experiments were carried out in both LB and M63/glucose media and no substantial difference was observed regarding plasmid positioning and plasmid foci number. We have also analysed RK2 mini-derivative localization in $P$. aeruginosa (data not shown). Using either TetR-EYFP tagging or FISH experiments we demonstrated that pCVI localization in $P$. aeruginosa is similar to that in $P$. putida.

In control experiments, the expression of tetR-eyfp in E. coli and P. putida cells without pCVI resulted in yellow fluorescence of the whole cells (Fig. 1C ii, H ii). When tetR-eyfp was not expressed in the cells, no signal was detected (Fig. 1Ci, H i). Also FISH and DAPI co-staining demonstrated no signal when bacterial cells did not contain pCVI (Fig. 1C iii, H iii).

\section{P. putida ParB protein interacts with RK2 DNA by binding centromere-like sites}

The fluorescence microscopy experiments indicated that the subcellular localization of an RK2 mini-derivative that lacks partitioning genes depends on the host bacterium.
This raised the possibility that the subcellular position of an RK2 mini-derivative could be affected by host factors, including the host-encoded partitioning machinery. Some similarities between the RK2 and Pseudomonas partitioning systems have been described (Hayes, 2000). In addition, RK2 and Pseudomonas, but not E. coli, have a type I par locus (Mohl \& Gober, 1997; Ogasawara \& Yoshikawa, 1992). The ParB protein from P. putida shows high similarity to the RK2 KorB protein (Lin \& Grossman, 1998): based on amino acid sequence, it is $27 \%$ identical with KorB. It has also been demonstrated that KorB of RK2 specifically interacts with the $P$. putida chromosomal $c$ is site (Chiu \& Thomas, 2004). We asked if the P. putida ParB protein could interact with RK2 DNA. Size-exclusion chromatography was used to investigate this possibility. Purified ParB protein was incubated with or without plasmid DNA and then fractionated using a Sepharose CL$4 \mathrm{~B}$ column. The results demonstrated that $P$. putida ParB formed nucleoprotein complexes with RK2 DNA (Fig. 2). When the entire RK2 plasmid or its mini-derivative pCVI were present in the incubation mixture, the ParB protein was detected in the column void volume, indicating the 
formation of a nucleoprotein complex. In the absence of DNA or in the presence of pUC18 DNA, no ParB protein was found in void volume fractions (Fig. 2). This result indicated interaction of ParB with RK2 plasmid DNA. The pCVI plasmid used in our study did not contain inc $C$ or the korB genes; however, two RK2 centromere-like sites, $\mathrm{O}_{\mathrm{B}}{ }^{10}$ and $\mathrm{O}_{\mathrm{B}}{ }^{3}$, were still present adjacent to the $\operatorname{trf} A$ and oriT sequences. To investigate whether those sites are capable of interacting with $P$. putida ParB protein, DNA fragments containing $\mathrm{O}_{\mathrm{B}}{ }^{10}, \mathrm{O}_{\mathrm{B}}{ }^{3}$ or $P$. putida centromere site IR2 were introduced into pUC18 plasmid DNA. When incubations were carried out with these plasmid constructs, ParB was detected in the void fractions (Fig. 2). Since no ParB complex formation was observed with pUC18, we concluded that the observed nucleoprotein complexes are the result of ParB protein interaction with RK2 centromere-like sites. The RK2 $\mathrm{O}_{\mathrm{B}}$ motifs differ from the Pseudomonas IR2 sequence; therefore we could not exclude the possibility that ParB possesses two domains for interaction with DNA.

To test the possibility that this interaction also occurs in $P$. putida cells, a chromatin immunoprecipitation with polyclonal anti-ParB antibodies followed by a PCR reaction with $\mathrm{O}_{\mathrm{B}}{ }^{3}-, \mathrm{O}_{\mathrm{B}}{ }^{10}$-, oriV- or IR2-specific oligonucleotide primers was performed. The interaction of the ParB protein with RK2 $\mathrm{O}_{\mathrm{B}}{ }^{3}$ and P. putida chromosomal IR2 was clearly detected when RK2 mini-derivative pCVI (Fig. 3A, B, lane 1 and Fig. 4 lane 1) was present in P. putida cells. Less intensive bands of $\mathrm{O}_{\mathrm{B}}{ }^{10}$-specific PCR product detected in immunoprecipitation experiments suggest that ParB interaction with this site on RK2 mini-derivatives could be less efficient (Fig. $3 \mathrm{~A}$, lane 1 and Fig. 4 lane 1). When ori $V$-specific primers were used, we did not detect any signal, indicating that a ParB complex is not formed with the RK2 origin (Fig. 3A, lane 1 and Fig. 4 lane 1).When the RK2 mini-derivative was not present in $P$. putida cells, we were able to detect ParB interaction only with the chromosomal IR2 sequence (Fig. 3B, lane 2). Similar results indicating $P$. putida ParB interaction with both $\mathrm{O}_{\mathrm{B}}{ }^{3}$ and $\mathrm{O}_{\mathrm{B}}{ }^{10}$ sites were obtained when the entire RK2 plasmid was present in $P$. putida cells (Fig. 3C lane 2). When RK2 plasmid was present in E. coli cells, no signal was detected, indicating that anti-ParB antibodies did not interact with RK2 KorB protein (Fig. 3C lane 3). These data showed that in P. putida cells chromosomally encoded ParB protein interacts with centromere-like $\mathrm{O}_{\mathrm{B}}$ sites of plasmid RK2 DNA.

\section{P. putida ParAB affects the position of RK2 replicons}

To further explore the possibility that the Pseudomonas partitioning machinery could affect the subcellular position of RK2 mini-derivatives, we investigated whether the expression of $P$. putida par $A B$ could change the asymmetrical polar distribution of the RK2 mini-derivative in E. coli cells. Plasmid pLAK15 containing both the gene for TetREYFP and $P$. putida parAB under the control of the $\mathrm{P}_{\mathrm{BAD}}$

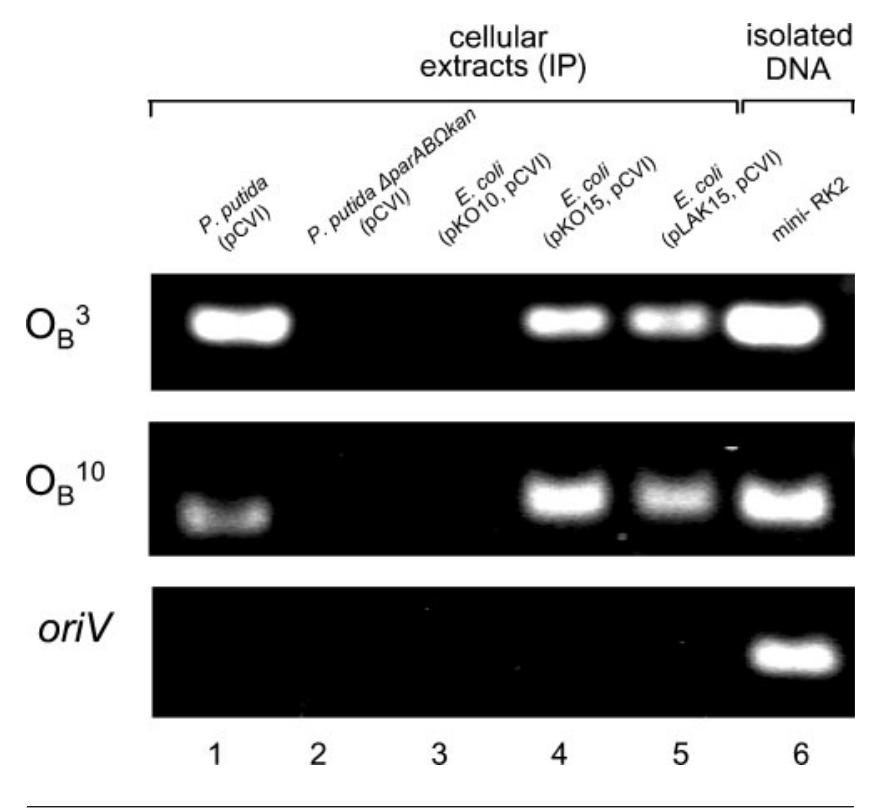

Fig. 4. $P$. putida ParB protein interacts in live E. coli S17-1 cells with centromere-like sites of the RK2 mini-derivative. PCRamplified products of immunoprecipitation (IP) with anti-ParB antibodies and lysates of E. coli S17-1(pCVI, pKO10) (lane 3), E. coli S17-1 (pCVI, pKO15) (lane 4) or E. coli S17-1 (pCVI, pLAK15) (lane 5). pKO15 and pLAK15 are vectors for expressing $P$. putida parAB. pKO10 is a pLAK15 derivative which does not contain $P$. putida parAB (see Table 1). Lanes 1, 2 and 6 , control of immunoprecipitation with anti-ParB antibodies and lysates of $P$. putida KT2440(pCVI) (lane 1) and P. putida KT2440 $\Delta$ parAB $\Omega k a n$ (pCVl) (lane 2), and control reactions with isolated DNA (lane 6) on $1 \%$ agarose gels stained with ethidium bromide. Primers used in reactions were designed for RK2 plasmid sequences containing $\mathrm{O}_{\mathrm{B}}{ }^{3}, \mathrm{O}_{\mathrm{B}}{ }^{10}$ and oriV regions. All primer sequences are listed in Methods.

promoter was constructed and introduced into E. coli cells containing the RK2 mini-derivative pCVI. In control experiments, in E. coli cells, instead of pLAK15 we used pKO10, which expresses only tetR-eyfp. After arabinose induction, when only the tetR-eyfp gene was expressed, the pCVI localization was polar and asymmetric (Fig. 5A-C). Approximately $40 \%$ of the cells contained fluorescent plasmid foci and multiple signals were detected in only $3 \%$ of the cells (Fig. 5M). In contrast, when TetR-EYFP and both ParA and ParB P. putida proteins were expressed, the distribution of pCVI in E. coli cells was more or less symmetrical, with plasmid foci located in the proximity of the mid-cell or the $1 / 4,3 / 4$ positions (Fig. 5D-F). Also, the proportion of the cells containing plasmid foci increased (Fig. 5M). We detected plasmid foci in $60 \%$ of the cells and among them approximately $30 \%$ had more than one fluorescent signal. These data were very similar to those obtained in a control experiment where pCVI number and location were analysed in wild-type P. putida cells (Fig. 5G-I, $\mathrm{M}, \mathrm{N}$ ). Further, we asked if $P$. putida ParB protein interacted with pCVI centromere-like sites when both ParA and ParB 

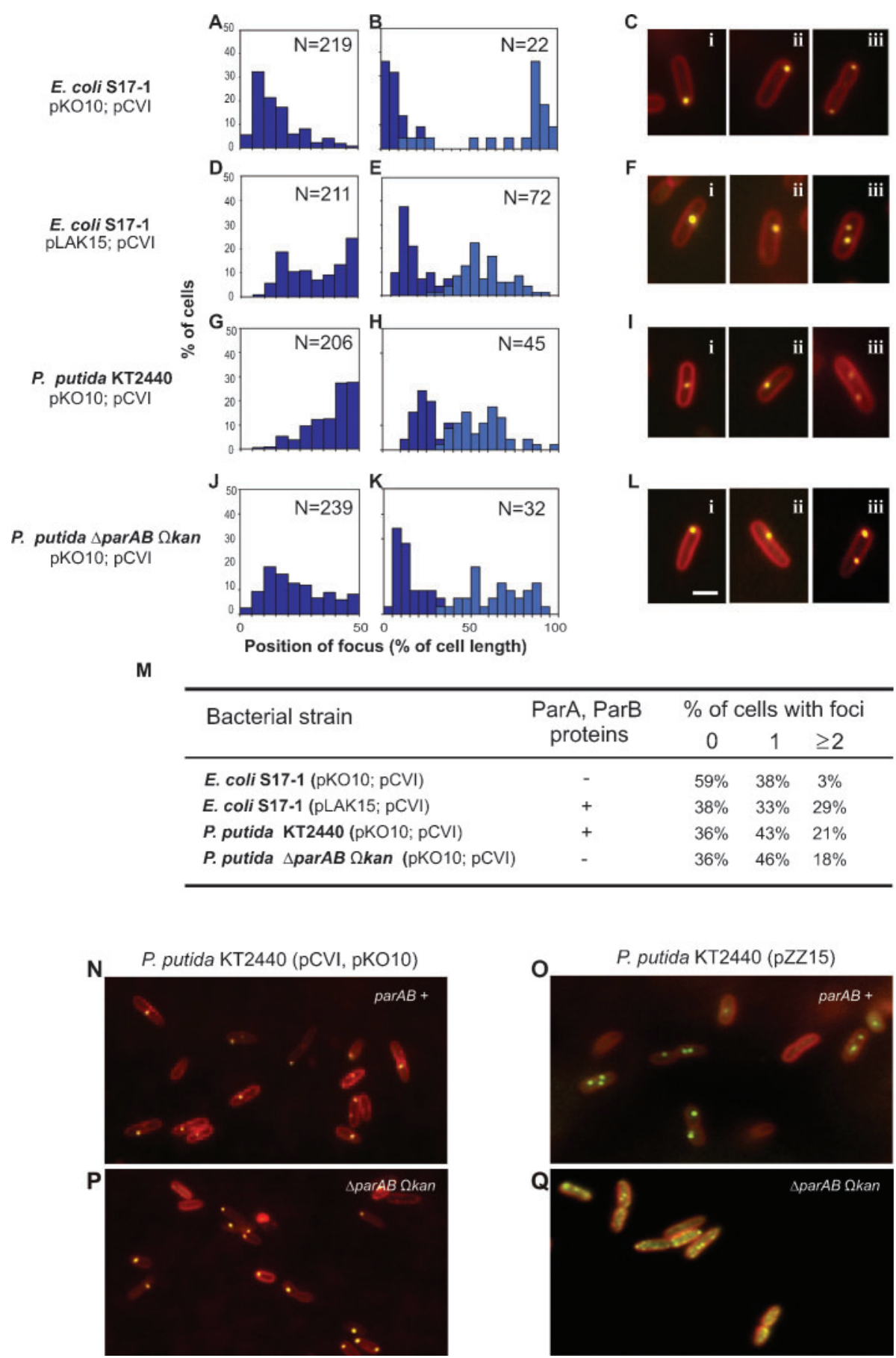

Fig. 5. $P a r A$ and $P a r B$ proteins from $P$. putida alter the location of RK2 plasmid. The subcellular position of $p C V I(A-L, N, P)$ was determined by tagging with TetR-EYFP in E. coli S17-1 (A-F), P. putida KT2440 (G-I, N) and P. putida KT2440 $\triangle$ parAB $\Omega$ kan (J-L, P). The subcellular position of pZZ15 was determined by tagging with Lacl-GFP in P. putida KT2440 (O) and $P$. putida KT2440 $\triangle$ parABskan (Q). Bacteria were grown in LB medium at $30{ }^{\circ} \mathrm{C}$. Genes for TetR-EYFP, ParA and ParB were expressed from pLAK15, whereas only tetR-eyfp was expressed from pKO10 and lacl-gfp from pZZ15. Cell membranes were stained with FM4-64 (red). Representative cells with one (i, ii) and two (iii) foci of pCVI are shown in panels $C, F, I$ and $L$. Panels A, D, G and J demonstrate the subcellular distribution of pCVI for cells containing one focus. In order to facilitate comparison, histograms have been used in which the distance of the plasmid focus to the nearest cell pole is shown as a fraction of cell length. Panels B, E, H and K demonstrate subcellular distribution of mini-RK2 for cells with two foci. Cell length $(\mu \mathrm{m})$ is plotted against position of foci given as a fraction of cell length. (M) Table showing the percentage of cells containing no, one or two and more fluorescent foci when ParA and ParB proteins are or are not present in the cells. 
were expressed from pLAK15 or pKO15 in E. coli cells. Chromatin immunoprecipitation demonstrated that indeed under those experimental conditions promoting the midcell or the $1 / 4,3 / 4$ positions of pCVI in E. coli cells (Fig. 5DF), both $\mathrm{O}_{\mathrm{B}}{ }^{3}$ and $\mathrm{O}_{\mathrm{B}}{ }^{10}$ sites were bound by $P$. putida ParB (Fig. 4, lanes 4 and 5). In a control reaction, no PCR products were observed when $E$. coli cells contained plasmid pKO10, without the P. putida parAB region (Fig. 4, lane 3).

We also analysed the subcellular location of pCVI in a $P$.

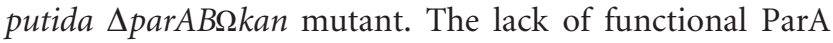
and ParB proteins changed the localization of the pCVI foci from the approximately mid-cell and $1 / 4,3 / 4$ positions to positions near the cell poles (Fig. 5J-L, P). We did not observe differences in the number of cells containing plasmid foci; however, the number of cells with a single signal was slightly increased compared to the wild-type $P$. putida (Fig. 5M). No $\mathrm{O}_{\mathrm{B}}{ }^{3}$ - and $\mathrm{O}_{\mathrm{B}}{ }^{10}$-specific PCR products were observed after immunoprecipitation of $P$. putida $\triangle \operatorname{par} A B \Omega k a n(\mathrm{pCVI})$ extract with anti-ParB antibodies (Fig. 4, lane 2). In the control, when ori $V$-specific primers were used (Fig. 4, lane 2), no signal was detected either.

Interestingly, when the localization of the entire RK2 plasmid (pZZ15) was analysed in the $P$. putida

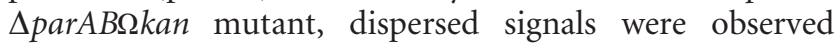
compared with the localization of pZZ15 in P. putida wild-type cells (Fig. 5O, Q). This indicates that in P. putida disturbances in the chromosomally encoded partitioning system affect the subcellular position of RK2 plasmid.

\section{Stability of RK2 and its mini-derivative pCVI in $E$. coli and $P$. putida strains}

The data presented above clearly show that the expression of $P$. putida parAB genes affects the position of RK2 mini-replicons. In E. coli, the expression of $P$. putida par $A B$ genes compensates for the lack of the RK2 partitioning system with regard to the cellular location of the RK2 miniderivative pCVI. In P. putida, the lack of chromosomal partitioning genes results in a shift of the position of pCVI towards the cell poles and disturbances of the localization of the entire RK2 plasmid. To test if the observed subcellular localizations of the RK2 plasmids affect their stabilities, we analysed the maintenance of pZZ15 and pCVI in the strains used in the course of this work. pZZ15 was stably maintained in both E. coli S17-1 and P. putida KT2440 (Fig. 6A, E). Although we observed disturbances in

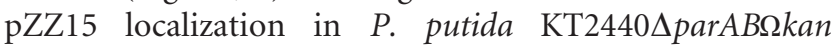
(Fig. 5Q), stability tests revealed that the plasmid is stably maintained in this mutant (Fig. 6A). This result could be explained by the compensating effect of the dispersed plasmid localization. Stabilities of RK2 mini-derivative pCVI in E. coli S17-1 and P. putida KT2440 were significantly reduced compared to the stabilities of pZZ15 in these strains (compare Fig. 6E with Fig. 6A and B). It must be pointed out that pCVI stability in P. putida KT2440, where the plasmid was found at approximately mid-cell and the $1 / 4,3 / 4$ positions (Fig. 1G), was better when compared to pCVI stability in E. coli S17-1, where it was located at the cell poles (Fig. 1B and compare Fig. 6E and $\mathrm{B}$ ). The disruption of the $P$. putida parAB locus

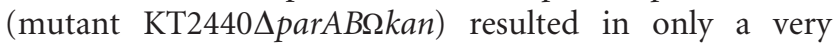
limited reduction of pCVI stability (Fig. 6B). A similar effect was observed when pCVI stability was tested with pKO10 also present in the cell. The pCVI stability observed

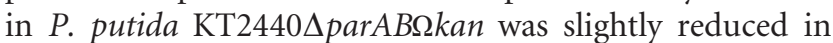
comparison with its stability in $P$. putida KT2440 (Fig. 6C). Similarly, only a limited effect on chromosome stability

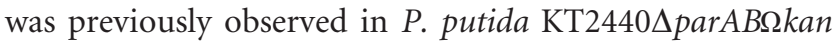
(Godfrin-Estevenon et al., 2002). Also, during our experiments the pCVI stability loss rate tested in E. coli in the presence of $\mathrm{pKO} 15$ or $\mathrm{pKO} 10$ was very similar, regardless of the expression of $P$. putida parAB genes (Fig. 6D). However, at the beginning of the experiment more cells contained pCVI in the strain where $P$. putida parAB was expressed (Fig. 6D).
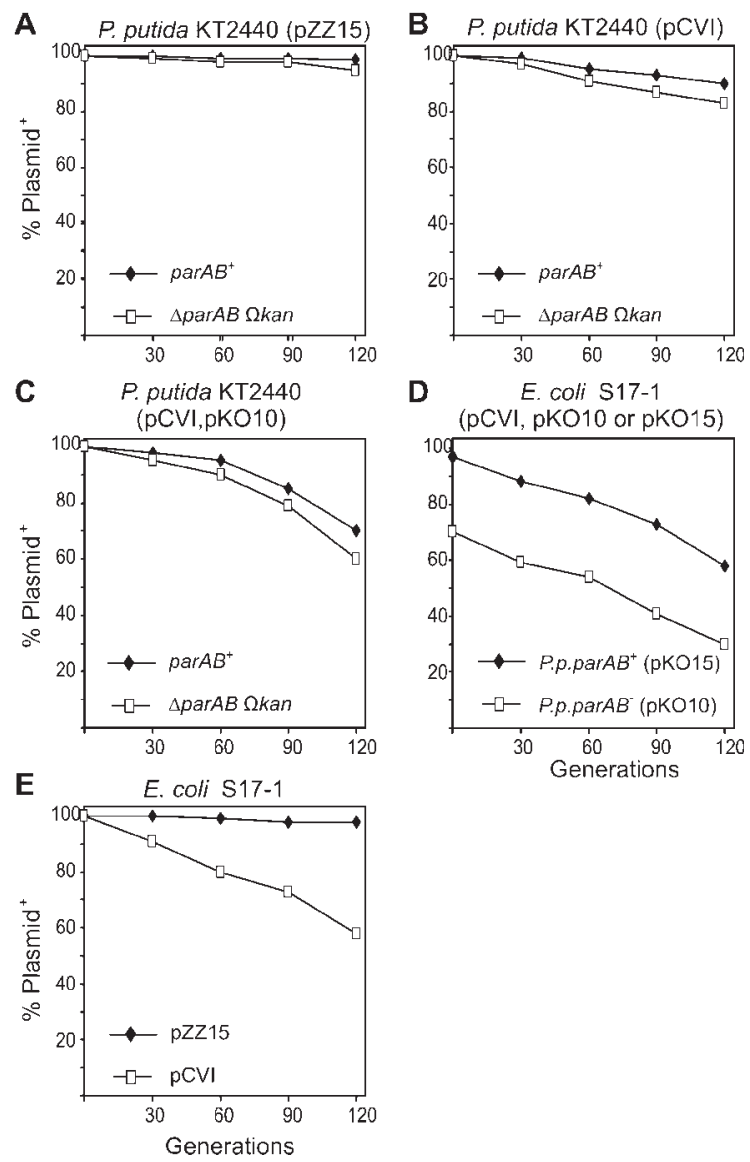

Fig. 6. Stability of $\mathrm{pCVI}$ and $\mathrm{pZZ15}$ in E. coli S17-1, $P$. putida KT2440 and $P$. putida KT2440 $\Delta$ parAB $\Omega$ kan cells. Stability assays were performed as described in Methods. Stability of pZZ15 in $E$. coli (E) and $P$. putida (A). Stability of $p C V I$ when the mini-derivative was present alone in the cells $(B, E)$ or in the presence of another plasmid, pKO10 or pKO15, as indicated (C, D). 
A limited effect of the parAB locus on RK2 mini-derivative stability could be a result of ParA and ParB, which may not support plasmid segregation as efficiently as IncC and KorB. It is also possible that the full stabilization of the plasmid mini-derivatives requires other factors such as additional $\mathrm{O}_{\mathrm{B}}$ sites, or post-segregation killing ( $p s k$ ) and multimer resolution ( $m s r$ ) systems that are missing in RK2 mini-derivatives. Accordingly, when the RK2 par locus containing korB and incC genes was introduced into pCVI DNA, it restored the mid- and quarter-cell positions of the plasmid but did not stabilize the plasmid during cell growth (Verheust \& Helinski, 2007).

In summary, the experiments reported here demonstrate, we believe for the first time, that the subcellular position of broad-host-range plasmid RK2 is affected by Pseudomonas partitioning machinery. The Pseudomonas partitioning module plays a role in chromosomal segregation (Godfrin-Estevenon et al., 2002; Lewis et al., 2002); however, it has also been speculated that it is a remnant of RK2-like plasmid integration (Chiu \& Thomas, 2004). That may explain cross-talk between chromosomal and plasmid partitioning systems. Our results at least raise the possibility that in certain bacteria the chromosomally encoded partitioning machinery could affect the subcellular positioning of a specific plasmid element.

\section{ACKNOWLEDGEMENTS}

We are grateful to Christopher Thomas for providing anti-ParB antibodies, to Donald Helinski and David Sherratt for providing pCVI and pLAU plasmids and to David Lane for supplying the strain

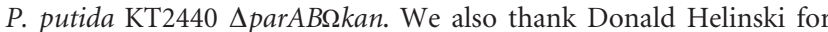
critically reading the manuscript. This work was supported by the Polish State Committee for Scientific Research Grant 2P04A02730, The Foundation for Polish Science and the EMBO/HHMI Young Investigator Programme. Katarzyna Kolatka and Marcin Pierechod are recipients of stipends from the Foundation for Polish Science.

\section{REFERENCES}

Chiu, C. M. \& Thomas, C. M. (2004). Evidence for past integration of IncP-1 plasmids into bacterial chromosomes. FEMS Microbiol Lett 241, 163-169.

Davis, M. A. \& Austin, S. J. (1988). Recognition of the P1 plasmid centromere analog involves binding of the $\operatorname{ParB}$ protein and is modified by a specific host factor. EMBO J 7, 1881-1888.

Ebersbach, G. \& Gerdes, K. (2004). Bacterial mitosis: partitioning protein ParA oscillates in spiral-shaped structures and positions plasmids at mid-cell. Mol Microbiol 52, 385-398.

Ebersbach, G. \& Gerdes, K. (2005). Plasmid segregation mechanisms. Annu Rev Genet 39, 453-479.

Ebersbach, G., Ringgaard, S., Moller-Jensen, J., Wang, Q., Sherratt, D. J. \& Gerdes, K. (2006). Regular cellular distribution of plasmids by oscillating and filament-forming ParA ATPase of plasmid pB171. Mol Microbiol 61, 1428-1442.

Franklin, F. C., Bagdasarian, M., Bagdasarian, M. M. \& Timmis, K. N. (1981). Molecular and functional analysis of the TOL plasmid pWWO from Pseudomonas putida and cloning of genes for the entire regulated aromatic ring meta cleavage pathway. Proc Natl Acad Sci U S A 78, 7458-7462.

Funnell, B. E. (2005). Partition-mediated plasmid pairing. Plasmid 53, 119-125.

Godfrin-Estevenon, A. M., Pasta, F. \& Lane, D. (2002). The parAB gene products of Pseudomonas putida exhibit partition activity in both P. putida and Escherichia coli. Mol Microbiol 43, 39-49.

Gordon, G. S., Sitnikov, D., Webb, C. D., Teleman, A., Straight, A., Losick, R., Murray, A. W. \& Wright, A. (1997). Chromosome and low copy plasmid segregation in E. coli: visual evidence for distinct mechanisms. Cell 90, 1113-1121.

Gordon, S., Rech, J., Lane, D. \& Wright, A. (2004). Kinetics of plasmid segregation in Escherichia coli. Mol Microbiol 51, 461-469.

Hayes, F. (2000). The partition system of multidrug resistance plasmid TP228 includes a novel protein that epitomizes an evolutionarily distinct subgroup of the ParA superfamily. Mol Microbiol 37, 528-541.

Ho, T. Q., Zhong, Z., Aung, S. \& Pogliano, J. (2002). Compatible bacterial plasmids are targeted to independent cellular locations in Escherichia coli. EMBO J 21, 1864-1872.

Isaac, J. H. \& Holloway, B. W. (1968). Control of pyrimidine biosynthesis in Pseudomonas aeruginosa. J Bacteriol 96, 1732-1741.

Jensen, R. B. \& Gerdes, K. (1999). Mechanism of DNA segregation in prokaryotes: ParM partitioning protein of plasmid R1 co-localizes with its replicon during the cell cycle. EMBO J 18, 4076-4084.

Jensen, R. B. \& Shapiro, L. (1999). Chromosome segregation during the prokaryotic cell division cycle. Curr Opin Cell Biol 11, 726-731.

Konieczny, I. \& Helinski, D. R. (1997). Helicase delivery and activation by DnaA and TrfA proteins during the initiation of replication of the broad host range plasmid RK2. J Biol Chem 272, 33312-33318.

Kostelidou, K. \& Thomas, C. M. (2000). The hierarchy of KorB binding at its 12 binding sites on the broad-host-range plasmid RK2 and modulation of this binding by IncC1 protein. J Mol Biol 295, 411-422.

Kovach, M. E., Elzer, P. H., Hill, D. S., Robertson, G. T., Farris, M. A., Roop, R. M. \& Peterson, K. M. (1995). Four new derivatives of the broad-host-range cloning vector pBBR1MCS, carrying different antibiotic-resistance cassettes. Gene 166, 175-176.

Lau, I. F., Filipe, S. R., Soballe, B., Okstad, O. A., Barre, F. X. \& Sherratt, D. J. (2003). Spatial and temporal organization of replicating Escherichia coli chromosomes. Mol Microbiol 49, 731-743.

Lawley, T. D. \& Taylor, D. E. (2003). Characterization of the doublepartitioning modules of R27: correlating plasmid stability with plasmid localization. J Bacteriol 185, 3060-3067.

Lewis, R. A., Bignell, C. R., Zeng, W., Jones, A. C. \& Thomas, C. M. (2002). Chromosome loss from par mutants of Pseudomonas putida depends on growth medium and phase of growth. Microbiology 148, 537-548.

Li, Y. \& Austin, S. (2002). The P1 plasmid in action: time-lapse photomicroscopy reveals some unexpected aspects of plasmid partition. Plasmid 48, 174-178.

Lin, D. C. \& Grossman, A. D. (1998). Identification and characterization of a bacterial chromosome partitioning site. Cell 92, 675-685.

MacKenzie, C., Chidambaram, M., Sodergren, E. J., Kaplan, S. \& Weinstock, G. M. (1995). DNA repair mutants of Rhodobacter sphaeroides. J Bacteriol 177, 3027-3035.

Meyer, R., Figurski, D. \& Helinski, D. R. (1975). Molecular vehicle properties of the broad host range plasmid RK2. Science 190, 12261228. 
Mohl, D. A. \& Gober, J. W. (1997). Cell cycle-dependent polar localization of chromosome partitioning proteins in Caulobacter crescentus. Cell 88, 675-684.

Niki, H. \& Hiraga, S. (1997). Subcellular distribution of actively partitioning F plasmid during the cell division cycle in E. coli. Cell 90, 951-957.

Ogasawara, N. \& Yoshikawa, H. (1992). Genes and their organization in the replication origin region of the bacterial chromosome. $\mathrm{Mol}$ Microbiol 6, 629-634.

Pogliano, J., Ho, T. Q., Zhong, Z. \& Helinski, D. R. (2001). Multicopy plasmids are clustered and localized in Escherichia coli. Proc Natl Acad Sci U S A 98, 4486-4491.

Rosche, T. M., Siddique, A., Larsen, M. H. \& Figurski, D. H. (2000). Incompatibility protein IncC and global regulator $\mathrm{KorB}$ interact in active partition of promiscuous plasmid RK2. J Bacteriol 182, 60146026.

Sambrook, J., Fritsch, E. F. \& Maniatis, T. (1989). Molecular Cloning: a Laboratory Manual, 2nd edn. Cold Spring Harbor, NY: Cold Spring Harbor Laboratory.

Schumacher, M. A. \& Funnell, B. E. (2005). Structures of ParB bound to DNA reveal mechanism of partition complex formation. Nature 438, 516-519.
Thomas, C. M., Meyer, R. \& Helinski, D. R. (1980). Regions of broadhost-range plasmid RK2 which are essential for replication and maintenance. J Bacteriol 141, 213-222.

Thomas, C. M., Cross, M. A., Hussain, A. A. \& Smith, C. A. (1984). Analysis of copy number control elements in the region of the vegetative replication origin of the broad host range plasmid RK2. EMBO J 3, 57-63.

Verheust, C. \& Helinski, D. R. (2007). The incC korB region of RK2 repositions a mini-RK2 replicon in Escherichia coli. Plasmid 58, 195-204.

Weitao, T., Dasgupta, S. \& Nordstrom, K. (2000). Plasmid R1 is present as clusters in the cells of Escherichia coli. Plasmid 43, 200-204.

Williams, D. R., Macartney, D. P. \& Thomas, C. M. (1998). The partitioning activity of the RK2 central control region requires only inc $C$, korB and KorB-binding site $\mathrm{O}_{\mathrm{B}} 3$ but other KorB-binding sites form destabilizing complexes in the absence of $\mathrm{O}_{\mathrm{B}} 3$. Microbiology 144 , 3369-3378.

Wilson, J. W., Sia, E. A. \& Figurski, D. H. (1997). The kilE locus of promiscuous IncP $\alpha$ plasmid RK2 is required for stable maintenance in Pseudomonas aeruginosa. J Bacteriol 179, 2339-2347.

Edited by: L. Jannière 